\title{
EL EMPRESARIO CULTURAL: ESTUDIO EXPLORATORIO (CUALITATIVO) SOBRE EMPRESARISMO EN LAS ARTES
}

\begin{abstract}
JAVIER HERNÁNDEZ-ACOSTA
Universidad de Puerto Rico. Escuela Graduada de Administración de Empresas, Rio Piedras, Puerto Rico javihernandez@yahoo.com
\end{abstract}

VÍCTOR QUIÑONES Universidad de Puerto Rico. Escuela Graduada de Administración de Empresas, Rio Piedras, Puerto Rico victorqnns@gmail.com

\section{Resumen}

Este trabajo responde a tres preguntas sobre el emprendimiento cultural: iEs consciente el artistaemprendedor de las exigencias que tiene ser artista, emprendedor y administrador de su creación? ¿Qué motiva a un artista a emprender su propio negocio? ¿Se siente satisfacción al ser emprendedor a la par con la gestión artística o llega a ser un mal necesario? Un grupo de emprendedores culturales participó en un proyecto piloto orientado a conocer de manera detallada sus perspectivas sobre las interrogantes anteriores. Los investigadores dependimos de entrevistas profundas individuales para conocer dichas perspectivas. Sin pretender generalizar sus resultados, los autores entienden que este esfuerzo permite conocer a emprendedores de un sector que aporta hasta el 11\% del producto interno bruto y del empleo de algunas economías y que no siempre cuenta con apoyo público o privado para llevar a cabo su labor creativa.

PALABRAS CLAVE: EMPRENDIMIENTO, EMPRENDIMIENTO CULTURAL, GESTIÓN DE LAS ARTES, PERFIL DEL EMPRENDEDOR CULTURAL.

\section{ABSTRACT}

This research offers answers to three questions: Is the cultural entrepreneur aware of the demands associated to his/her activities both as an artist as well as an entrepreneur and as the manager of his/her own business? What motivates an artist to embrace entrepreneurship? Does an artist feel satisfied with both roles, as an artist and as an entrepreneur? A pilot study was conducted among a group of cultural entrepreneurs to find answers to these questions based on individual in-depth interviews. Although findings are not to be applied to all cultural entrepreneurs, it is expected that this study could bring a major understanding of a sector that contributes up to $11 \%$ of the Gross Domestic Product and to employment within economic activities that are not always supported by either the public or private sectors.

KEY WORDS: ENTREPRENEURSHIP, CULTURAL ENTREPRENEURSHIP, MANAGING THE ARTS, PROFILE OF THE CULTURAL ENTREPRENEUR.

\section{INTRODUCCIÓN}

Hasta hace algunos años economía y cultura parecían ser términos que no tenían espacio en una misma oración. Sin embargo, desde que Adorno y Horkenheimer (1947) utilizaron por primera vez el término industria cultural en el contexto del surgimiento de medio de reproducción masiva y homogeneizadora de las expresiones culturales, como respuesta a las tendencias económicas de la época, ha comenzado el interés en la investigación sobre el impacto que esta industria puede tener en el desarrollo económico de los países (UNCTAD, 2004, Quartesan et al., 2007; Finger, 2006;y Getino, 2000). La nueva vertiente debe ser estudiada como el análisis de la generación de valor económico, o sea, un complemento, y no un sustituto, de la importancia del valor estético, social y cultural que este tipo de actividad genera, y que, en última instancia, es el principal motiva- 
dor de su producción. A partir del desarrollo de las industrias culturales, diversas organizaciones internacionales como la "United Nations Education for Science and Culture Organization" (UNESCO), la "United Nations Comission on Trade and Development" (UNCTAD), el "World Intellectual Property Organization" (OMPI, por sus siglas en español) y el Banco Interamericano de Desarrollo (BID) han realizado extensos estudios sobre la elaboración de estadísticas e indicadores culturales, el impacto del sector cultural en el empleo, el Producto Interno Bruto (PIB) y el fomento de industrias culturales como mecanismo para reducir la pobreza en los países en desarrollo (Finger, 2006; UNCTAD, 2004, 2008).

La UNESCO (2006) define la industria cultural como "aquellos sectores relacionados con la creación, la producción y la comercialización de contenidos que son inmateriales y culturales en su naturaleza. Típicamente, estos sectores son protegidos por el derecho de autor y pueden tomar la forma de bienes y servicios". Lo anterior incluye, entre otros sectores, manifestaciones como las artes visuales, editoriales, plásticas, escénicas y musicales. Según el economista David Throsby (2001), existen tres características que definen las industrias culturales: (1) La creatividad como elemento principal de su producción, (2) la generación y comunicación de significados simbólicos y (3) que posean en sus productos finales algún tipo de propiedad intelectual. En los últimos años, y con los cambios que han provocado los avances tecnológicos, se ha comenzado a utilizar el término industrias creativas, categoría dentro de la cual se engloban sectores como las artes gráficas, la publicidad y el diseño de software, entre otros (Garnham, 2005).

\section{La empresa cultural}

Al igual que en el caso de empresas que sirven otros propósitos, las que pertenecen a la industria cultural también deben contar con la financiación y estructura organizativa necesaria para su gestión. Aunque existen distintos modelos organizacionales para estructurar la empresa cultural, sobre todo las relacionadas con actividades artísticas como las promovidas por músicos, pintores y actores, entre otros, resulta común que aquellos que amplían sus horizontes más allá de la inmediatez de su arte, ejerzan los papeles de emprendedor y de administrador de estas. Ello se debe a que, en muchos casos, las empresas creadas carecen de recursos para emplear personas con peritaje, sobretodo en áreas administrativas.

En realidad, la industria cultural cuenta con un alto nivel de auto-empleo (Menger, 1999): el artista emprendedor crea su empresa, la administra y se compensa con los recursos generados por la actividad que lleva a cabo. Pero, al igual que en otros sectores económicos, quizá de manera más pronunciada en la empresa cultural, esta gestión no siempre garantiza un flujo continuo de ingresos con los que el emprendedor-administrador pueda depender para vivir y hacer crecer su empresa. Por esta misma razón, encontramos que una parte sustancial de la actividad cultural no se genera por las dinámicas del libre mercado, si no debido al subsidio de entidades gubernamentales o de algunas instituciones privadas y del tercer sector, que surgen con el fin de apoyarlas. Aunque en demasiadas ocasiones, el artista permanece toda su carrera profesional como emprendedor y administrador, en un pequeño número de casos, el nivel de reconocimiento y "éxito" llega a un punto en el cual comienza a delegar estas funciones mediante la subcontratación o, inclusive, pasando a ser empleado de una empresa dedicada a la producción artística. Lo anterior lleva a preguntarse lo siguiente: ¿Es consciente el artista-emprendedor de las exigencias que tiene ser artista, emprendedor y administrador de su creación? ¿Qué motiva a un artista a emprender su propio negocio? Estas son las preguntas de un proyecto de investigación que motivaron el presente estudio exploratorio llevado a cabo entre un grupo de emprendedores-administradores-. 
A continuación se explica la manera en que se estructuró este artículo. La segunda sección expone la aportación que hacen las empresas culturales a las economías nacionales y la pertinencia del empresarismo en dicho sector. También se incluyen algunos de los problemas confrontados por aquellos que deciden involucrarse en el llamado empresarismo cultural.

La tercera sección recoge los resultados de una serie de entrevistas a profundidad llevadas a cabo entre un grupo de artistas-emprendedores-administradores que permite conocer las motivaciones, los momentos álgidos de la gestión empresarial-administrativa, los logros alcanzados y sus perspectivas sobre el desarrollo de su persona como empresario y de la organización que dirigen.

\section{MARCO TEÓRICO \\ Organizaciones Culturales}

Modelos para medir el impacto

de las organizaciones culturales

Ya a principios de la década de 1980, la UNESCO comenzó a reconocer la importancia del sector cultural en el desarrollo económico de los países. A pesar de los intentos de la UNESCO en desarrollar un sistema uniforme de medición de estadísticas en las industrias culturales a través del globo, han surgido varios modelos cuyo uso depende del país donde se utilicen.

En particular, el Modelo de los Círculos Concéntricos, elaborado por Throsby (2008b), plantea como eje principal la creación artística, a partir de la cual se extienden círculos que se van alejando del centro en la medida en que su contenido va perdiendo valor cultural y acercándose más al valor económico (Véase Figura 1).

FIGURA 1

\section{FÓRMULA DE CÍRCULOS CONCÉNTRICOS EN LAS INDUSTRIAS CULTURALES}

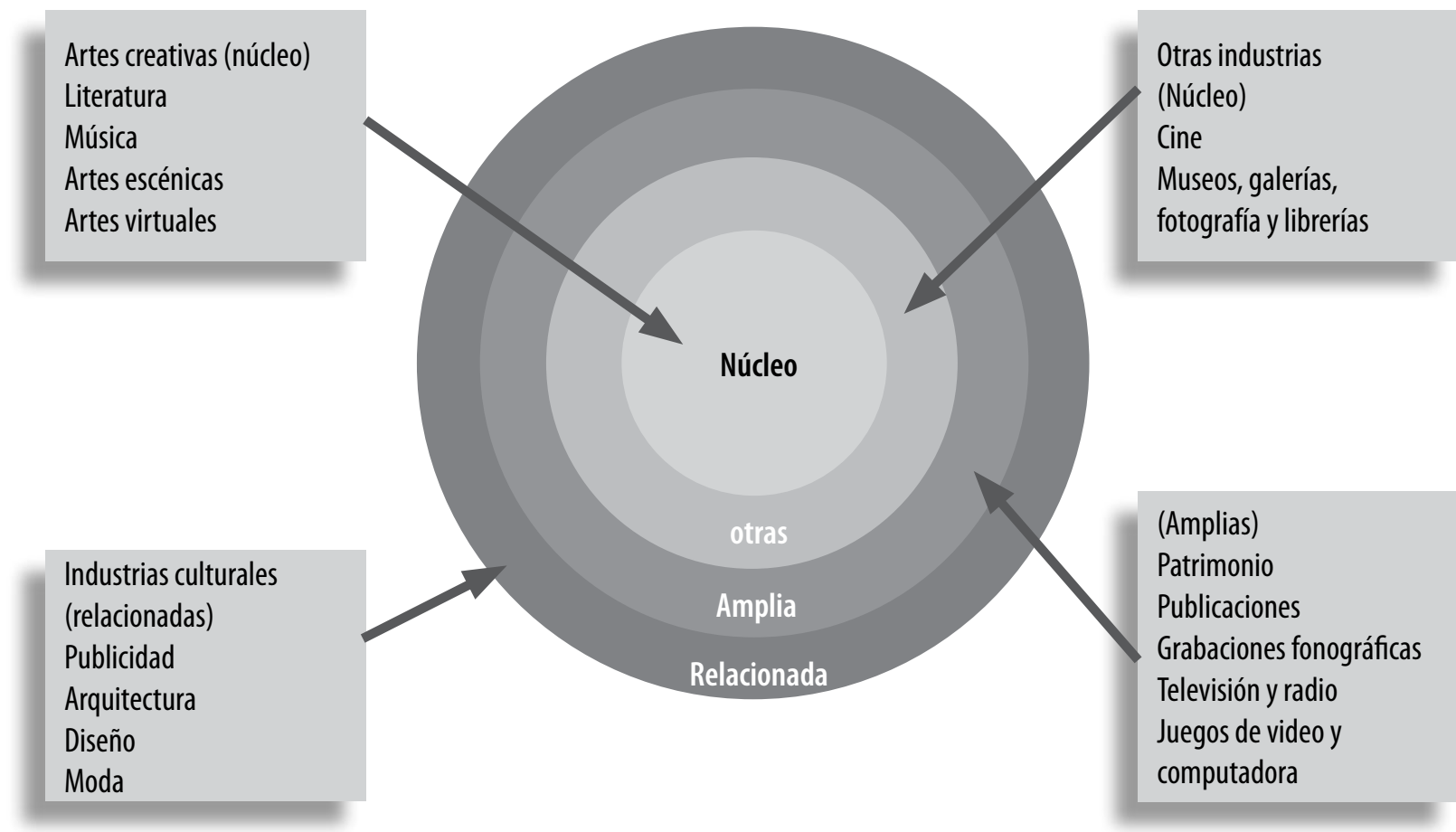

Fuente: David Throsby, The Concentric Circles Model of the Cultural Industries, 2008. 
Otros modelos, como el de la Organización Mundial para la Propiedad Intelectual (OMPI, por sus siglas en inglés), se basan únicamente en la capacidad de generar ganancias de la propiedad intelectual, mientras que el modelo de "Contenidos Simbólicos" se concentra principalmente en el valor cultural.

En medio del análisis que busca entender el impacto de las industrias culturales en el desarrollo de las economías nacionales, es preciso incluir las distintas dimensiones de valor que genera este tipo de actividad. Para John Holden, (2004) el problema radica en la dificultad de medir el influjo de las formas de valor no-económicas porque la relación de causa y efecto es indirecta y subjetiva. Sin embargo, Holden plantea la importancia de aplicar análisis antropológicos en términos de valor histórico, social, simbólico, estético y espiritual. De igual forma, sugiere la sostenibilidad en términos de asegurar el disfrute de las futuras generaciones, la pre- caución a los daños irreversibles y una adaptación de la biodiversidad como mecanismo para asegurarse de que las distintas manifestaciones culturales nunca desaparezcan permanentemente.

\section{El impacto económico de las organizaciones culturales}

A través de la utilización de los distintos modelos presentados, se han comenzado a establecer estimados del impacto económico de las organizaciones culturales en el desarrollo de los países. Según la UNCTAD (2004), estas representan un $7 \%$ de Producto Interno Bruto (PIB) mundial y se estima que su crecimiento puede ser a razón de un 10\% anual (UNCTAD, 2004). Durante 2003, en Inglaterra, las organizaciones culturales generaron ingresos de $£ 110$ billones y emplearon a 1.3 millones de personas (Depto. de Cultura, Medios y Deportes, UK, 2003, citado en UNCTAD, 2004).

TABLA 1

\section{CONTRIBUCIÓN AL PIB Y EL EMPLEO DE LAS INDUSTRIAS CULTURALES (SEGÚN METODOLOGÍA OMPI)}

\begin{tabular}{|c|c|c|c|c|c|}
\hline País & Año del Estudio & \% PIB (Total) & \% PIB (Industrias Núcleo) & \% Empleo (Total) & \% Empleo (Industrias Núcleo) \\
\hline Bulgaria & 2005 & $2,81 \%$ & $1,57 \%$ & $4,30 \%$ & $2,29 \%$ \\
\hline Jamaica & 2005 & $4,81 \%$ & $1,70 \%$ & $3,03 \%$ & $1,79 \%$ \\
\hline Líbano & 2005 & $4,75 \%$ & $2,53 \%$ & $4,49 \%$ & $2,11 \%$ \\
\hline México & 2003 & $4,77 \%$ & $1,55 \%$ & $11,01 \%$ & $3,41 \%$ \\
\hline Filipinas & 1999 & $4,82 \%$ & $3,50 \%$ & $11,10 \%$ & $8,81 \%$ \\
\hline Canadá & 2004 & $4,70 \%$ & $3,50 \%$ & $5,40 \%$ & $4,00 \%$ \\
\hline Hungría & 2002 & $6,66 \%$ & $3,96 \%$ & $7,10 \%$ & $4,15 \%$ \\
\hline Letonia & 2000 & $5,05 \%$ & $2,90 \%$ & $5,59 \%$ & $3,70 \%$ \\
\hline Singapur & 2001 & $5,67 \%$ & $2,85 \%$ & $5,80 \%$ & $3,64 \%$ \\
\hline USA & 2004 & $11,09 \%$ & $6,48 \%$ & $8,53 \%$ & $4,07 \%$ \\
\hline Australia & 2007 & $10,30 \%$ & $7,30 \%$ & $8,00 \%$ & $4,97 \%$ \\
\hline Croacia & 2004 & $4,27 \%$ & $2,99 \%$ & $4,64 \%$ & $3,22 \%$ \\
\hline Rumania & 2005 & $5,55 \%$ & $3,55 \%$ & $4,19 \%$ & $2,36 \%$ \\
\hline Colombia & 2005 & $3,30 \%$ & $1,90 \%$ & $5,80 \%$ & $1,70 \%$ \\
\hline Rusia & 2004 & $6,06 \%$ & $2,39 \%$ & $7,30 \%$ & $4,29 \%$ \\
\hline Ucrania & 2005 & $2,85 \%$ & $1,54 \%$ & $1,90 \%$ & $1,16 \%$ \\
\hline Holanda & 2005 & $5,90 \%$ & $4,00 \%$ & $8,80 \%$ & $6,20 \%$ \\
\hline
\end{tabular}

*Fuente: OMPI, 2010 www.wipo.int

Fuente: Organización Mundial para la Propiedad Intelectual, 2010. 
En Latinoamérica, los países del Mercosur y Chile, fueron parte de un estudio sobre el impacto de las organizaciones culturales relacionadas con el derecho de autor, utilizando la metodología de la OMPI. Dicho estudio determinó que en Brasil, Argentina y Uruguay, la participación del sector cultural en el PIB promedió un 6\%, mientras que en Chile y Paraguay se encontraba entre el $1 \%$ y $2 \%$. Por su parte, el empleo generado por dicho sector se encontraba entre el $3 \%$ y el $5 \%$ en los cincos países investigados (Marcio, 2002).

La Organización Mundial para la Propiedad Intelectual (OMPI) también ha promovido investigaciones similares en distintos países, de donde surgen los datos sobre el impacto en el PIB y el Empleo que se presenta en la Tabla 1.

\section{FIGURA 2}

\section{CONTRIBUCIÓN AL PIB DE LAS EMPRESAS CULTURALES (SEGÚN METODOLOGÍA OMPI)}

\% PIB (Total) $\square$ \% PIB (Industrias Núcleo)

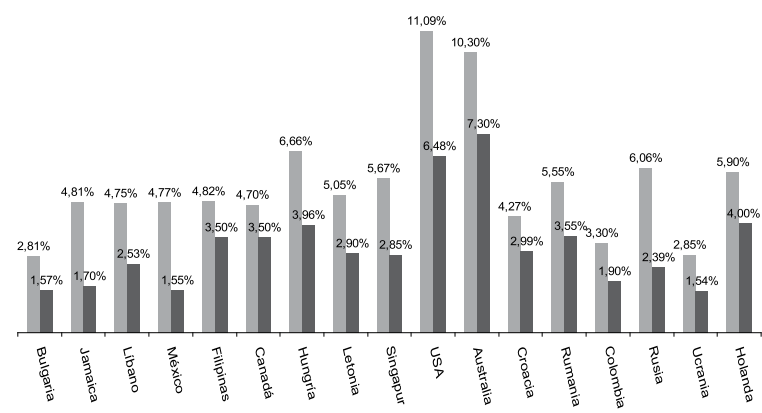

Figura 3

\section{CONTRIBUCIÓN AL EMPLEO DE LAS INDUSTRIAS CULTURALES (SEGÚN METODOLOGÍA OMPI)}

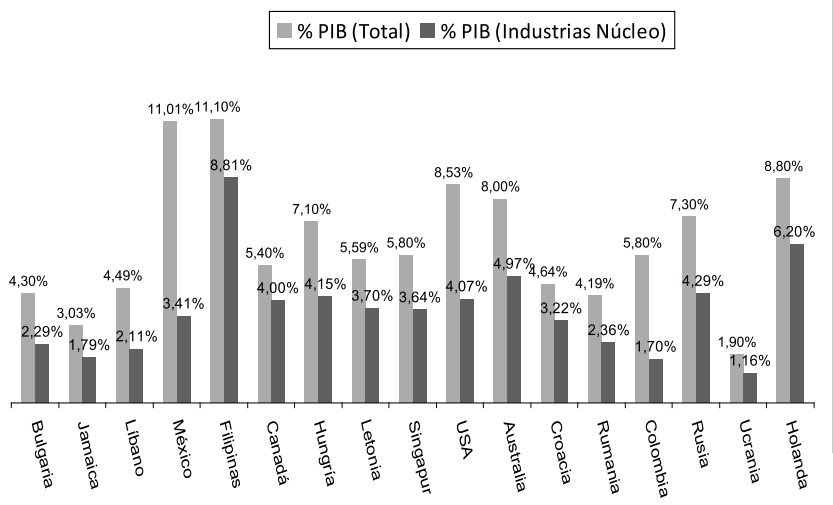

En las figura 3 se presenta la contribución total al empleo y el PIB y también la contribución de las organizaciones que representan el núcleo, según el modelo, ya que son estas las que incluyen a los emprendedores culturales. Estos datos reflejan el impacto directo de este sector económico en los países. La comparación entre las organizaciones núcleo y el total (incluyendo organizaciones de apoyo) reflejan en qué segmento de la cadena de valor se concentra esta contribución. Casos como Estados Unidos y Australia demuestran un gran impacto en la parte de creación, mientras que casos como México, muestran una gran contribución al empleo, pero principalmente en otros segmentos que no son los de creación.

Además de la contribución económica en el nivel macroeconómico, las organizaciones culturales tienen un gran impacto en el desarrollo local. Entre los planteamientos de beneficios se encuentran su intensidad en mano de obra, ser respetuosas con el ambiente, estar interconectadas entre ciudades y regiones, estar basadas en conocimientos locales tácitos, su contribución a mejorar la calidad de vida y su papel crítico en la promoción de la cohesión social y de sentido de pertenencia (Quartesan et al., 2007). La literatura también resalta la capacidad del sector en reflejar y construir valores para la sociedad, lo que representa un vehículo de identidad cultural (Quartesan et al., 2007).

\section{Características y problemas de las organizaciones orientadas a las artes}

Las organizaciones culturales, en especial aquellas relacionadas con la creación artística como la música, el teatro y las artes plásticas, tienen características que pueden afectar grandemente el desempeño de los emprendedores en los distintos sectores. En el documento Arte y Aparte: Manual para el emprendimiento en artes 
e industrias creativas, creado por el Ministerio de Cultura de Colombia (2005), se discuten varios mitos que afectan el fomento de estos emprendimientos. Entre estos se encuentran que los artistas no deben involucrarse en la gestión administrativa, que los productos culturales no se pueden medir por resultados, que existe un alto riesgo para los artistas en relacionarse con otros agentes y que el trabajo artístico es exclusivamente individual. Todos estos mitos cuentan con argumentos en contra que demuestran la capacidad de emprender y de ser exitosas de las empresas artísticas, si se entienden la naturaleza y los procesos de comercialización de los bienes culturales (Min. Cult. Colombia, 2005). Otras perspectivas que enfrenta el desarrollo de los artistas como emprendedores son: la percepción romántica de estos (conocida como el arte por el arte) que elimina el interés económico de los artistas; la cultura como un bien público y de libre acceso; y el cuestionamiento sobre la capacidad del mercado y de los individuos para definir valor monetario y no solamente de los "expertos" (Hesmondhalgh \& Pratt, 2005).

Ya en el siglo XVIII, Adam Smith había considerado que el trabajo de los artistas no era productivo, ni contribuía a la "riqueza de las naciones" porque "su trabajo perece al momento mismo de su producción" (citado por Prieto, 2001 en Asuaga et al., 2005). Esto supone grandes dificultades para estimar la demanda de estos productos y servicios (Ministerio de Cultura Colombia, 2005) y que la satisfacción hacia un "producto" cultural no garantiza su mismo nivel en un momento posterior (Caves, 2000).

En términos de producción, los costos iniciales son muy altos, limitando el desarrollo de este tipo de proyectos por el alto riesgo que representan (Garnham, 2005). Sin embargo, los costos marginales son pocos o ningunos, permitiendo el desarrollo de economías de escala, lo que hace que la rentabilidad del proyecto dependa de la cantidad de unidades (o eventos) que se puedan producir y vender (Asuaga et al., 2005). Según Hesmondhalgh \& Pratt (2005), los grandes conglomerados de la industria utilizan a las pequeñas empresas como vehículos de investigación y desarrollo, dejando caer sobre estos toda la vulnerabilidad que conlleva probar la rentabilidad de un proyecto cultural. Los casos que demuestran rentabilidad son aceptados por los conglomerados, quienes a través de su capacidad económica para la producción y el mercado, se benefician de economías de escala.

En términos de mercadeo del producto cultural, se presenta como limitación, en algunos sectores, la inelasticidad de la demanda al cambio en precio (Asuaga et al., 2005; Colbert, 2003). Un ejemplo en cuanto a la inelasticidad de demanda de los productos culturales es el caso de los museos: típicamente, su asistencia no aumenta aún siendo libres de pago, lo que implica que existen otros factores que motivan el consumo cultural, algo que representa un reto para el artista. Francois Colbert (2003) plantea que es muy difícil aumentar la cantidad de consumidores culturales porque se depende, en gran medida, de la actitud que se genere hacia los productos culturales desde los años de infancia. Por lo tanto, ante la gran cantidad y amenaza de productos substitutos (Asuaga et al., 2005), resulta necesario desarrollar una estrategia de segmentación y posicionamiento que permita a los emprendedores culturales diferenciarse de competidores en un mercado sobresaturado [cuando de ocio y entretenimiento se trata] (Colbert, 2003). Además de estas estrategias, es importante que los emprendedores y organizaciones artísticas desarrollen enfoques de investigación en torno a la conducta del cliente, en torno a la distribución, a la promoción y al desarrollo de productos (Finn et al., 1994). De forma final, debemos mencionar que contrario a lo que plantean los mercadólogos: comenzar con la identificación de deseos en los consumidores, a ser satisfechos mediante el desarrollo de productos, la mayoría de los bienes y servicios culturales se comportan de la 
siguiente manera: primero se produce el bien o servicio y luego se identifica un mercado para este. La perspectiva de los mercadólogos podría ser contraria a la creatividad que caracteriza la génesis del producto cultural típico, por lo que resulta dudosa su implantación cuando de artistas se trata. A su vez, su rechazo trae como consecuencia los fracasos de comercialización de un número de productos culturales y la frustración de sus creadores.

\section{Emprendimiento}

Se ha comprobado que existe una figura dentro del sistema económico, primordialmente capitalista, que identifica y explota oportunidades, basándose en la intuición y la información, y que está rodeada de elementos de incertidumbre y riesgo: el emprendedor. La palabra emprendedor comenzó a utilizarse en el Siglo XIII, proveniente del término "entreprendre" que significa "hacer algo" o "llevar a cabo", y ya para el siglo XVI se utilizaba directamente para referirse a aquel que desarrollaba un nuevo negocio (Hall \& Sobel, 2006).

Aunque la descripción de quién es el emprendedor ha dependido de diferentes enfoques teóricos, expuestos a lo largo de los años, se puede establecer que al menos se trata de alguien que descubre, evalúa y explota un oportunidad de crear un nuevo producto o servicio (Shane \& Venkatamaran, 2000). A pesar de que dicha descripción no es estrictamente producto de los economistas, pues su contenido revela que distintas disciplinas han aportado misma esta, no cabe duda de que los estudiosos de la teoría económica, así como de disciplinas próximas a la economía, han hecho importantes contribuciones sobre la figura del emprendedor.

Los psicólogos, por otro lado, sugieren que el emprendedor es una persona con enfoque hacia las metas, la auto-confianza y el riesgo, entre otras características personales, aunque de igual forma plantean que estas características implica- rían que el individuo siempre es un emprendedor y no solamente en un momento dado (Ripsas, 1998). Otros sugieren que el emprendedor es en realidad un artista cuya creatividad -y no el capital- es el verdadero secreto del éxito de su gestión empresarial (Faltin \& Zimmer, 1995, citado en Ripsas, 1998).

\section{El perfil del artista-emprendedor}

El mercado laboral de los artistas se caracteriza por fuentes tradicionales de empleo como el trabajo a tiempo parcial, segundas ocupaciones, altos niveles de auto-empleo y desempleo, contrataciones temporeras y una edad promedio menor que la de la fuerza laboral general (Benhamau, 2003; Menger, 1999). Una característica importante de los artistas es que parecen estar dispuestos a conseguir segundos empleos a tiempo parcial en trabajos de remuneración baja para complementar el ingreso de los trabajos artísticos. Esto sugiere que los beneficios no pecuniarios de su profesión compensan la pérdida por el salario no generado (Papandrea \& Albon, 2004), algo que es consistente con los hallazgos de Hamilton (2000) sobre la diferencia en productividad y los beneficios no-económicos. Una perspectiva similar es la descrita como el "arts pool" o banco de artistas, que sugiere que estos prefieren tener trabajos de remuneración baja y poco compromiso, que les permita abandonarlo en cuanto surjan oportunidades relacionadas con la profesión artística. Esta conducta se repetirá constantemente a lo largo de la carrera del artista (Papandrea \& Albon, 2004).

Son pocos los artistas que pueden trabajar a tiempo completo en su profesión. A pesar de los pocos datos disponibles en los distintos países, en el caso de Australia, por ejemplo, solo el 11\% de los artistas trabajan exclusivamente en una profesión directamente relacionada con la parte creativa de las artes. Si se incluye la educación en las artes, la cifra aumenta a un 57\% (Papandrea \& Albon, 2004). Sobre este aspecto, el economista 
William Baumol hace una comparación entre los artistas y los investigadores académicos, proponiendo que ambos sostienen su actividad principal a través de la educación (Baumol, citado en Menger 1999). De igual forma, en el caso de Australia, uno de cada tres artistas, tiene ingresos por debajo de los niveles de pobreza (Throsby \& Hollister, 2003). El 25\% de los participantes en un estudio manifestó haber estado desempleado durante un periodo promedio de 17 meses en algún momento de los últimos cinco años ( $\mathrm{Pa}$ pandrea \& Albon, 2004).

En el caso de Estados Unidos, en el año 2005, 1.99 millones de habitantes identificaron las artes como su primera profesión. Según un estudio, estas personas poseen un nivel de educación mayor a la fuerza laboral y son 3.5 veces más propensos al auto-empleo que el resto de la población (National Endowment for the Arts, 2008). El estudio del National Endowment for the Arts (NEA), también encontró que, en promedio, los artistas generan $\$ 6,000$ menos anualmente que otros profesionales. A pesar de las complejas condiciones de trabajo, el empleo en el sector se mantiene creciendo al mismo ritmo que el total de la fuerza laboral (NEA, 2008 y Thorsby \& Hollister, 2003).

Precisamente, uno de los problemas más citados en el análisis económico de las artes es el exceso de oferta de artistas. Esta situación puede estar motivada por las ganancias y reconocimiento extraordinario de unos pocos (Rosen, 1981), lo que convierte el empleo en las artes como una lotería en un mercado de talento especulativo (Menger, 1999). Sin embargo, en los últimos años, los adelantos tecnológicos han reducido los requerimientos para desempeñarse en este sector, aumentando la producción y la oferta de artistas (Menger, 1999).

El auto-empleo es actualmente la forma de trabajo más frecuente para los artistas (Menger, 1999), lo que supone un alto nivel de emprendimiento. Si examinamos las características que normalmente se le atribuyen a los emprendedores, encontrare- mos que muchas de ellas coinciden con la de los artistas. La capacidad de crear valor a través de la producción de trabajos para la venta, un gran compromiso y productividad, asociada a la independencia ocupacional, control sobre su trabajo, flexibilidad, independencia, sentido de orientación hacia el desempeño y el alto riesgo, son algunas de estas cualidades (Menger, 1999 \& Ellmeier, 2003). Quizá lo que los distinga es la pasión que muestran los artistas en su esfuerzo, bastante independiente de compensaciones financieras.

Eikhof \& Haunschild (2006) destacan las cualidades de lo que describen como el emprendedor bohemio. Lo describen como alguien cuya vida privada está subordinada al trabajo y evaden grandes compromisos financieros como tener una casa o seguros, con el fin de tener movilidad para cumplir con los compromisos de trabajo. Están conscientes de su responsabilidad sobre mercadearse continuamente y acostumbran a tener una relación menos jerárquica con sus superiores, en este caso directores y productores. A pesar de que el trabajo creativo surge en muchas ocasiones individualmente, tienen un gran sentido del colectivo y gran disposición para trabajar en equipo (Wilson \& Stokes, 2002).

Al igual que en el caso de los emprendedores, los artistas parecen tener un nivel de satisfacción muy alto con su ocupación (Menger, 1999), algo que podría estar relacionado con el nivel de autonomía, iniciativa, poca rutina, capacidad de utilizar destrezas múltiples en el trabajo, gran sentido de comunidad y oportunidad de reconocimiento social.

Sin embargo, a pesar de que estas características emprendedoras pueden representar una oportunidad para el desarrollo de una industria cultural competitiva, aún se requiere que los empresarios culturales desarrollen otras destrezas como conocer de promociones, de comunicación efectiva y de contar con la autosuficiencia financiera que les permita optimizar sus condiciones de intercambio en su gestión empresarial. 
Finalmente, resulta interesante la observación de Andrea Ellmeier (2003), quien propone que estas experiencias de los emprendedores culturales, además de contribuir a acortar la distancia entre el empleado y el emprendedor, ayudan a crear la cultura del "individuo emprendedor": "quienes no siguen las normas establecidas sino que intentan sus propias combinaciones para ubicarse en el mercado y la sociedad".

\section{METODOLOGÍA}

\section{Población}

La población de interés para la investigación sobre emprendimiento cultural estuvo integrada por personas residentes en Puerto Rico, encargadas, tanto de la producción del bien o servicio cultural, como de la administración y mercadeo de dicha gestión. Estas personas generan más de un $50 \%$ de su ingreso de la actividad cultural a la que se dedican y la describieron como su ocupación principal. Los sectores culturales incluidos fueron las artes visuales, editoriales, musicales y escénicas. Los participantes fueron contactados por uno de los investigadores a través de las redes sociales, tipo Facebook, cuya página los identificaba como emprendedores, y mediante amigos que conocían emprendedores. Por tanto, la muestra se seleccionó a conveniencia de los investigadores, dada la ausencia de un listado de emprendedores culturales localizados en Puerto Rico. La cantidad total de participantes, cuyas respuestas sirvieron para explicar a profundidad los temas planteados durante el diálogo, fue de 6 personas. Unas 16 personas fueron originalmente contactadas para participar del proyecto piloto. Varios de ellos declinaron de momento participar en la investigación por compromisos previos, por no contar con el tiempo que exigía la entrevista (entre 2 y 3 horas) o de momento sus respuestas fueron consideradas vagas o limitadas en contenido. Se espera que un mayor número de empresarios participe cuando el estudio sea ampliado.

\section{Recopilación y análisis de los datos}

La recopilación de datos dependió de entrevistas a profundidad individuales, que fueron grabadas y posteriormente transcritas. Las entrevistas tuvieron una duración de entre 2 y 3 horas. Se mantuvo la sugerencia de Gill Ereaut (2002) quien plantea que el investigador debe mantenerse interpretando resultados tanto a lo largo de la entrevista como posterior a esta, algo que solo puede llevarse a cabo cuando se trata de entrevistas a profundidad individuales o grupales (Ereaut, 2002). Para la consecución de las respuestas se utilizó una guía de temas relacionados con la motivación tenida para enfrascarse en la gestión cultural (convertirse en empresarios culturales), la experiencia vivida en dicha gestión y la evaluación del desempeño del emprendedor en dicha actividad. A continuación, se presenta el esquema usado para el análisis de los diálogos llevados a cabo durante el proceso:

\section{FIGURA 4}

\section{ESQUEMA DE TEMAS DE ANÁLISIS}

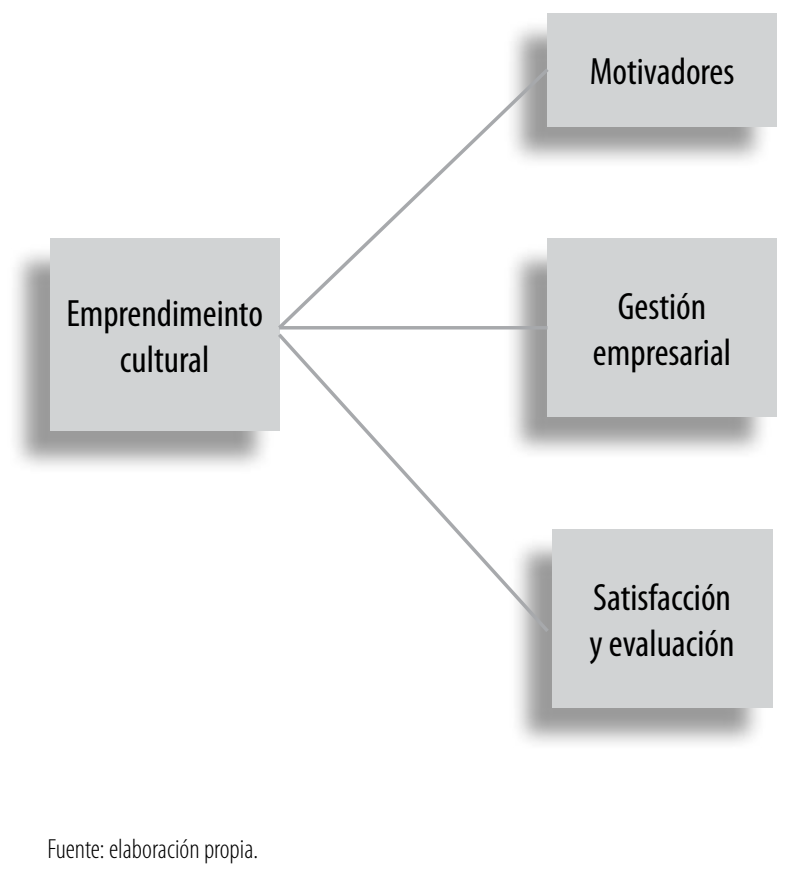




\section{RESULTADOS}

\section{Las motivaciones}

Las motivaciones para comenzar la actividad de emprendimiento en los sectores culturales parecen asociarse a la búsqueda y consecución de las siguientes metas (sin que medie un orden): la liberación de situaciones en las que abunda el trato injusto a los artistas, al ser subalternos de terceros, y que algunos consideran los hace sentirse "estúpidos" por tener que seguir el libreto impuesto, o sea, que el emprendimiento representa el momento ..."de hacerse dueño de su destino"; la puesta en marcha de esquemas innovadores (Shumpeter) que permitan hacer "...lo que se quiere hacer y no aquello que deja dinero, pero no gusta", y, el interés en desarrollar y difundir una propuesta artística independiente de terceros, que el funcionamiento del sector cultural no viabiliza. El aspecto del funcionamiento del sector merece ampliarse porque parece ser algo agobiante.

Según el estudioso de la Universidad de Harvard, Richard Caves, típicamente existe una brecha entre la demanda y la oferta por los productos culturales. El artista se inclina por su creación, independientemente de la existencia de demanda por esta. Pero, surgen los intermediarios para facilitar la selección de productos culturales que cuentan con el apoyo de los mercados (Caves, 2000). Sin embargo, al tratarse de "...personas típicamente ajenas al arte..." (los intermediarios) tienden a llevar a cabo un análisis muy subjetivo sobre qué puede o no ser aceptado por el público (audiencia). Por tanto, algunos emprendedores alegan que se trata de un recurso improductivo dentro de la cadena de valor de las industrias culturales. Los emprendedores consideran que lo mejor es, pues, prescindir de intermediarios. Lo más razonable para los emprendedores es "...eliminarlos del panorama...", y establecer sus propias maneras de desarrollo y difusión artística para así dar marcha al proyecto cultural de interés.

Lo expresado muestra más disgusto circunstancial que chispa natural interna para emprender la actividad cultural de interés. Pero, lo cierto es que "...esperar a que lleguen las oportunidades..." que, sin lugar a dudas, contengan la semilla del éxito, no es la manera de resolver lo que molesta o angustia. Por tanto, también "... hay que provocar", porque el artista debe ser "... un provocador..." de nuevas circunstancias. Voluntad y entusiasmo parecen ser dos vocablos asociados al emprendimiento (García, Álvarez \& Reyna, 2007) provocadores de los que deciden embarcarse en dicha tarea, independientemente de las oportunidades identificadas o de los vínculos con intermediarios. Incluso, si hay que ser "barman" a la par con la gestión empresarial "...que así sea..." pues "..en demasiadas ocasiones es imposible, en los comienzos, depender de la nueva empresa".

Vale mencionar que el acceso a la internet, así como a otros adelantos tecnológicos, sin dudas, ha ayudado a concretar que dicha provocación empresarial sea algo más que palabra, al convertirse en medio colaborador del emprendedor cultural. Internet, en particular, permite la difusión de la actividad creativa de forma rápida, amplia, menos costosa, así como eliminar la dependencia, si hay alguna, de intermediarios. Para algunos emprendedores, la tecnología aludida ayuda a educar a los mercados sobre sus esquemas innovadores, a encontrar "nichos" de audiencias que esperan "otra cosa" de sus artistas $y$, por ende, a permitir que las "perspectivas individuales del artista" logren espacios difíciles o imposibles de encontrar sin medios, entre otros, como internet, donde abundan las redes sociales que permiten experimentar y recibir inmediata reacción del público. Colbert (1994), en su momento, como hemos indicado, evaluaba esto de ofrecer lo que no necesariamente contaba con demanda, como la diferencia entre merca- 
dear arte y cultura y cualquier otro producto ya que, típicamente, en este último caso, la identificación de la demanda precede a la comercialización de la oferta. Parece que esa diferencia en el siglo XXI, aunque persiste, ya no representa el riesgo de décadas previas.

\section{La gestión empresarial-administrativa}

"La locura tiene que ser parte de la empresa...." pero, algunos emprendedores no se enteran de la "locura" que han comenzado a "padecer" hasta que descubren, sobre la marcha, su ignorancia del tema y la magnitud del esfuerzo y el tiempo que requieren las actividades administrativas. Hay quien evalúa la actividad administrativa como "arma de doble filo" pues permite "...el control de las cosas..." pero, a su vez, quita tiempo a la actividad creativa. No obstante, aunque para algunos emprendedores el aprendizaje administrativo es autodidacta, la propia experiencia, dada su vinculación previa al sector cultural en el que se involucran como gestores, les permite entender su funcionamiento y no comenzar a aprenderlo desde cero. Pero, quede claro que aun bajo circunstancias como las descritas, los esfuerzos de todo tipo, como es la planificación, la elaboración de un contenido estratégico, la gestión mercadológica así como la de financiación, siguen siendo aspectos con grandes retos, no siempre fáciles de confrontar y superar.

Es por ello que Bilton (2006) destaca la capacidad de los artistas para adaptarse a los cambios inesperados. Es que, pensar más allá de lo inmediato resulta un ejercicio difícil de plantearse. No es que los emprendedores en el sector cultural dejen de establecer metas a alcanzar en el largo plazo, sino que su consecución puede confrontar tantos escollos que bien vale funcionar a base de la "inmediatez" pues induce a trabajar "... de manera más eficiente....

Lo expresado no implica ausencia total de planificación por parte de los emprendedores. De hecho, existen situaciones en las que planificar el esfuerzo es tan pertinente como su presentación ante una audiencia. Por ejemplo, en las artes escénicas, artes visuales y artes editoriales, entre otras manifestaciones artísticas, la propia naturaleza de la producción requiere tiempo y esfuerzo para la investigación del producto que interesa comercializarse, para ensayos, para la identificación de recursos humanos y materiales, para la coordinación y para la promoción. En la música, los artistas planifican el momento de cada grabación y el tiempo que les deber tomar recuperar la inversión. De todas formas, en estos casos, el proceso de planificación no es más que una realidad ineludible. En general, se depende de la espontaneidad y de la capacidad para resolver los contratiempos de manera creativa.

Aún cuando la espontaneidad y la improvisación prevalecen en la gestión de la organización cultural, no es menos cierto que la mentalidad estratégica tiene su espacio entre algunos emprendedores. Esa mentalidad se refleja particularmente en momentos de mercadear productos que confrontan dificultades para romper bloques establecidos por grandes consorcios y sus representantes. Gracias a medios como internet, los emprendedores recurren directamente a los mercados para que estos conozcan y cuenten con la oferta creativa. Así se conocen inicialmente y así desarrollan, en parte, sus intercambios emprendedores y clientes.

Existen quienes, incluso, explotan servicios complementarios, como la adquisición y operación de estacionamientos y/o la venta de boletos vía medios electrónicos y telefónicos o hasta estableciendo estudios de grabación. En lugar de "... gastar el dinero sobrante de la actividad creativa en temas ajenos al negocio", se ahorra para adquirir equipos que permitan ampliar áreas que generan valor para los clientes (Gronroos, 2006).

El éxito alcanzado mediante acciones como las descritas puede deberse a lo que algunos 
estudiosos entienden como producto de un esfuerzo típicamente colectivo, casi gremial, de los integrantes de un grupo artístico. Lo anterior permite la continuidad o permanencia como grupo, dado que las estructuras jerárquicas son menos frecuentes entre ellos (Eikhof \& Haunschild, 2006, Wilson \& Stokes, 2002). Sin embargo, aún cuando se gestiona la participación de los integrantes del colectivo al momento de tomar decisiones, en ocasiones no es menos favorable para el colectivo el que haya un líder a quien recurrir tanto para "...dar seguridad al resto de la tripulación..." para resolver tranques, así como para "...echar culpas o achacar errores". Pero el colectivo sirve para delegar y evitarle desgastes al líder y también para la propia continuidad de la organización.

Como indicamos, en gran medida, el emprendedor cultural depende de su capacidad creativa tanto para el diseño y concreción del producto artístico, así como para las restantes actividades asociadas al esfuerzo de mercadear el mismo, tales como su difusión y distribución. No obstante, existe algo adicional por qué preocuparse: la marca.

En demasiadas ocasiones la marca del producto artístico, como en el caso de mercadeo de muchos servicios, se vincula con personas. Debido a dicha vinculación, cualquier esfuerzo orientado a dar a conocer el producto artístico es dar a conocer el nombre del artista creador. La marca es, pues, tanto reputación artística como personal. Su sostén y su desarrollo implican capitalizar para ambos.

Algunos emprendedores opinan que para establecer y solidificar su marca, algo que puede tomarles años y puede desaparecer en cuestión de segundos, deben trascender la relación "fan"artista, rompiendo barreras que existan entre ellos y la audiencia. Esto exige convertirse en parte de la comunidad a la que sirve el artista y hacer que los logros alcanzados por el artista sean también logros de la comunidad, sea esta local o global. Así, la identificación con situaciones que preocupan a la sociedad del lugar de origen del artista, así como aquellas que trascienden dicha frontera, forman parte del esfuerzo orientado hacia la consolidación de la marca del emprendedor.

Como en toda empresa que se inicia, no importa el sector, gestionar financiamiento es un reto formidable. Aquí también se demuestra la creatividad del emprendedor-artista. En sus inicios puede que se dependa de los recursos financieros personales del emprendedor, así como de la colaboración desinteresada de otros participantes (otros artistas que dan tiempo y esfuerzo sin cobrar salarios de momento o "...en lo que empresa echa hacia adelante"). Algunos dependen de fuentes externas como son los socios, amigos y parientes que creen en el proyecto del emprendedor y deciden apoyarlo. Otros, acuden a las instituciones financieras a solicitar prestamos personales con propósitos ajenos a la actividad cultural, pero el dinero servirá para lanzarla. Ejemplo de esta última situación es el caso de un emprendedor que pidió dinero a un banco para lanzar un disco, y, quien, además de encontrarse con la risa despectiva del oficial banquero, también encontró su negativa al préstamo. Esto no fue así cuando solicitó un nuevo préstamo para ampliar su cocina. Obviamente, utilizó el dinero del préstamo para su propósito inicial: lanzar su disco.

Si se logra comercializar el proyecto cultural surge otro reto ligado a los temas financieros: ¿cómo sostenerlo? Un área técnica que preocupa a los emprendedores es la de tarifar su oferta, pues los proyectos culturales no siempre cuentan con subsidios gubernamentales o privados, pero cuando cuentan con estos se espera que sean accesibles a aquellos clientes que los aprecian pero con limitada capacidad para patrocinarlos. 
Para los emprendedores tarifar es algo complejo y casi siempre fuera de su mejor capacidad de manejo. Si logran el apoyo de un contador que les asesore de manera gratuita, o por poco dinero, sienten que es cuestión de seguir sus sugerencias al pie de letra. De otro modo, procede "...hacer algún tipo de aritmética..., partiendo de los costos más obvios. De ser esta última la realidad con la que tienen que proceder, en más de una ocasión tienen que usar recursos personales para solventar el ejercicio creativo pues los errores de cálculo prevalecen. Lo cierto es que pocos emprendedores logran que su proyecto se prolongue por el tiempo necesario para recuperar la inversión inicial o para no considerarlos muertos al nacer. Algunos, más persistentes, gestionan auspicios de firmas comerciales que les sirvan para imprimir programas con los contenidos de la oferta creativa, costear la difusión de eventos o pagar alquileres de locales contratados, entre otras situaciones. Pero, siempre, "...con el agua al cuello". Ya los que pasan alguna temporada con relativo éxito, se sienten más capaces de pedir tarifas más adecuadas, que les permitan contar con sobrantes monetarios o ganancias. A medida que la marca gana adeptos y los espacios de oportunidades aumentan, queda atrás la preocupación de tarifar para no quedar con déficits.

\section{Autoevaluación de la gestión de emprendimiento}

De acuerdo con estudiosos del emprendimiento cultural, estos emprendedores típicamente sienten altos niveles de satisfacción con la gestión que llevan a cabo. Entre las razones para ello se encuentran aspectos tales como la autonomía y la variedad de destrezas que pueden utilizar en su gestión de negocios (Menger, 1999; Schjoedt, 2009). Para el emprendedor cultural basta estar dentro de dicho sector para sentirse "feliz, aún cuando la labor llevada a cabo no sea la de líder".
Son varias las razones encontradas para la satisfacción del emprendedor (satisfacción aquí definida como la coincidencia entre los resultados obtenidos al llevar a cabo la labor empresarial y las expectativas que se tenían al respecto). Tal como previamente se había indicado, una de estas razones es la autonomía o "flexibilidad que permite contar con el control de gran parte de tu tiempo y la libertad para hacerlo sin rendirle cuentas a un jefe...", así como el logro del balance entre la vida profesional, personal y familiar, logro que es producto de ese control del tiempo pero que a su vez implica que dicho balance es una meta deseable. También colabora con dicha satisfacción, poder retar la mente de las audiencias con propuestas no anticipadas por estas, gracias a la gestión creativa del emprendedor y su colectivo. Finalmente, el emprendedor se plantea su gestión como un proceso de aprendizaje continuo pues en cada día de esfuerzo acontecen situaciones inesperadas que ofrecen lecciones que desarrollan a la persona profesional y personalmente, además del reconocimiento de los demás por el trabajo llevado a cabo (mediante sus aplausos, sus críticas y su complicidad). Estos son resultados que permiten plantear satisfacción.

Sin embargo, no todo es satisfacción. Algunas áreas que provocan molestias, frustraciones e incluso deseos de abandono de proyectos incluyen: la ausencia de apoyo financiero para adelantarlos, el limitado o nulo apoyo de los mercados cuando se presentan propuestas innovadoras porque "nadas contra la corriente", la ausencia de destrezas administrativas y el propio hecho de estar aprendiendo sobre la marcha, lo que conduce a errores continuos que provocan insatisfacciones no siempre infrecuentes.

Pine y Gilmore (1999) expresaron que el "trabajo es un teatro y cada negocio un escenario". Resultarían, pues, aplicables a toda actividad de negocios los puntos generadores o no de satisfacción. Sin embargo, vale destacar que en el caso 
de los emprendedores culturales parece existir una pasión por la labor llevada a cabo, pocas veces observada entre emprendedores de otros sectores de negocios. El emprendedor cultural no parece involucrarse en dicha gestión necesariamente por evadir jerarquías o jefes, como en ocasiones acontece en los otros sectores; sí parecen hacerlo con el compromiso que generan los emprendedores de cultos religiosos. Para ellos su gestión es un "evangelio" que debe predicarse hasta donde lo permitan las fuerzas individuales. El empresario cultural es un apóstol dispuesto a todo, a cambio de su arte: pasa hambre si es necesario, incursiona en territorios poco amistosos y defiende su "doctrina", incluso a riesgo de afectar su persona. No es fácil que abandone su "culto" pues es su vida, aún cuando las condiciones no sean del todo propicias para continuar su evangelización. Allí estará siempre, dirigiendo su rebaño, acompañando los cómplices que forman su feligresía, porque también es seguidor de su propio evangelio.

\section{CONCLUSIONES}

En realidad sienten ser emprendedores en cuanto a que hacen algo por "su causa" evangelizadora en pro de la cultura, no en el sentido ideológico que impulsaría el sistema capitalista. De hecho, es el propio sistema el que, en general, los ha marginado al posicionarlos como no productivos, ya desde la época de Adam Smith. Por tanto, para el artista tomar el camino del empresarismo es probablemente "un mal necesario" ya que de otro modo no podrían impulsar y sostener su producto cultural. ¿Se sentirán raros o mal si desde afuera los llamamos emprendedores? Probablemente la respuesta sea un sí. Ellos son artistas que hacen labores administrativas necesarias que no les provoca pasión, complicidad o compromiso.

Eso sí, los emprendedores culturales cuentan con dosis de perseverancia, tolerancia al riesgo y a los retos, y mucha entrega al esfuerzo que llevan a cabo. Son apóstoles altamente comprometidos con "la doctrina predicada". En ellos la improvisación, la espontaneidad y la capacidad de responder a situaciones adversas con los recursos disponibles son realidades vitales que les permite sostenerse en los tiempos de cambio y rapidez que viven las economías mundiales. Quizá el propio hecho de sentirse siempre compartiendo como colegas con otros integrantes del sector cultural en el que se desempeñan, el de funcionar como un colectivo que cuenta con un líder, no con un jefe, haga que ese "mal necesario" sea menos oneroso y, por tanto, menos despreciado.

Algo que prevalece en el emprendimiento cultural es la mira a largo plazo, aún cuando parezca que se vive día a día. Ellos reconocen que lograr posicionarse como marca distinta toma esfuerzo y tiempo. Cada proyecto es, por ende, un escalón dentro de un largo caminar. Si ese largo caminar no ha generado más que la subsistencia, eso ya es bastante. Lo importante es que se incorporen más feligreses a su doctrina, más "fans" a su proyecto, más apoyo y reconocimiento a su esfuerzo. Ello ya es ganancia. Lo monetario será la consecuencia natural "...de un camino que se hace al andar".

"Una vez le pregunte a un productor: ¿Por qué tú haces teatro? Y me contestó porque le deja dinero. Le dije: pues sabes que yo no, lo hago porque las piezas me conmueven, y si me conmueven debe haber alguien a quien le puedan conmover... y también porque quiero aportar al desarrollo cultural de mi país. (Palabras de un emprendedor cultural.)

\section{Iniciativas de investigación futura}

Los investigadores son conscientes de que el contenido de los párrafos previos solo revela la 
punta del iceberg. Resulta prudente recalcar que ninguno de los hallazgos debe generalizarse hasta que se lleven estudios cuantitativos al respecto. De momento, los investigadores concentrarán sus esfuerzos en crear un marco de emprendedores culturales tanto puertorriqueños como del Caribe de habla española que les sirva a manera de un censo de dicha población. La creación del listado dependerá de nombres y organismos culturales provistos por instituciones tanto públicas como privadas de países como Puerto Rico, República Dominicana, Cuba, Venezuela y Colombia, entre otros. Una vez se logre crear el marco aludido los investigadores esperan llevar a cabo nuevos esfuerzos de investigación que partan de temas como los incluidos en el presente trabajo. Es nuestro interés elaborar el o los perfiles del emprendedor cultural del área caribeña de modo que otros investigadores, personas a cargo de la política cultural pública y otros interesados, particularmente donantes del sector privado, cuenten con el contenido necesario para ampliar el tema de emprendimiento cultural.

\section{Referencias}

Asuaga, C., Lecueder, M., \& Vigo, Silvia. (2005). The Performing Arts and the Cost General Theory. MPRA Paper No. 13742, Munich.

Benhamau, F. (2003). Artists' Labour Markets. Handbook of Cultural Economics, pp. 69-75, Massachusetts, USA: Edward Edgar Publishing.

Bilton, C. (2006). Cultures of Management: Cultural Policy, Cultural Management and Creative Organizations. Draft unpublished.

Caves, R. (2000). Creative Industries: Contracts between arts and commerce. Harvard Press, Cambridge, MA.

Casson, M. (1982). The entrepreneur: an economic theory. Edward Elgar Publishing, Cheltenham, UK.

Colbert, F. (2003). Entrepreneurship and Leadership in Marketing the Arts. International Journal of Arts Management, 6, 1, pp. 30-39.
Colbert, F. (1994). Marketing Culture and the Arts. Chair in Arts Management, HEC Press, Montreal (Quebec), Canada.

Eikhof, D., \& Haunchild, A. (2006). Lifestyle Meets Market: Bohemian Entrepreneurs in Creative Industries. Creativity and Innovation Management, 15, 3, pp. 234-241.

Finger, J. (2006). Poor People's Knowledge: Helping Poor People To Earn From Their Intellectual Property. Developmental Entrepreneurship: Adversity, Risk, and Isolation. International Research on the Business Disciplines, Volume 5, Elsevier, Oxford, UK.

Finn, A., McFadyen S., and Hoskins C. (1994). Marketing, Management, and Competitive Strategy in the Cultural Industries. Canadian Journal of Communication, Vol. 19, No 3. Recuperado el 29 de Julio de 2009 en http:// www.cjc-online.ca/index.php/journal/article/viewArticle/831/737

García, J., Alvarez, P., \& Reyna, R. (2007). Características del emprendedor de éxito en la creación de PYMES españolas. Estudios de Economía Aplicada, 25, 3, pp. 1-26.

Garnham, N. (2005). From Cultural to Creative Industries: An analysis of the implications of the "creative industries" approach to arts and media policy making in the United Kingdom. International Journal of Cultural Policy, 11,1, pp. 15-29.

Getino, O. (2000). Las Industrias Culturales en el Mercosur. Observatorio de Industrias Culturales de la Ciudad de Buenos Aires.

Gronroos, C. (2006). On Defining Marketing: Finding a New Roadmap For Marketing. Journal of Marketing Theory, 6,4, pp. 395-417.

Hall, J., \& Sobel, R. (2006). Public Policy and Entrepreneurship. Technical Report 06-0717, The Center for the Applied Economics. The University of Kansas, Lawren$\mathrm{ce}, \mathrm{KS}$.

Hamilton, B. (2000). Does Entrepreneurship Pay? An Empirical Analysis of the Returns to Self-Employment. Journal of Political Economy, 108, 3, pp. 604-631.

Hesmondhalgh, D. \& Pratt, A. (2005). Cultural Industries and Cultural Policy. International Journal of Cultural Policy, 11, 1, pp. 1-13.

Holden, J. (2004). Capturing Cultural Value: How culture has become a tool of government policy. Demos, London, UK. 
Horkheimer, M. \& Adorno, T. (1988). La industria cultural. Iluminismo como mistificación de masas. Dialéctica del Iluminismo. Sudamericana, Buenos Aires, Argentina.

Kirzner, I. (1999). Creativity and/or Alertness: A Reconsideration of the Schumpeterian Entrepreneur. Review of Austrian Economics, 11, pp. 5-17.

Marcio A. (2002). Estudio sobre la importancia económica de las industrias y actividades protegidas por el derecho de autor y los derechos conexos en los países del Mercosury Chile. Universidad Estadual de Campinas, Brasil.

Menger, P. (1999). Artistic Labor Markets and Careers. Annual Review Sociology, 25, pp. 541-574.

Ministerio de Cultura de Colombia. (2005). Arte y Aparte: Manual para el emprendimiento en artes e industrias creativas. Recuperado el 26 de junio de 2009 en http:// www.emprendimientosculturales.com/docs/49-artey-parte.

National Endowment for the Arts. Artist in the Workforce: 1990-2005. Research Report \# 48. NEA, Washington, DC.

Papandrea, F., \& Albon, R. (2004). A Model of Employment in the Arts. Blackwell Publishing Ltd, Australia.

Pine, B. Joseph \& Gilmore, James H. (1999). The Experience Economy. Harvard Business School Press, Boston.

Quartesan, A., Romis, M. \& Lanzafame, F. (2007). Las Industrias Culturales en América Latina y el Caribe: Desafíos y Oportunidades. Banco Interamericano de Desarrollo. Recuperado el 18 de junio de 2009 en http://www.scribd. com/doc/17484345/Industrias-Culturales-en-AmericaLatina-y-el-Caribe-Desafios-y-Oportunidades.
Ripsas, S. (1998). Towards an Interdisciplinary Theory of Entrepreneurship. Small Business Economics, 10, pp. 103115.

Rosen, S. (2001). The Economics of Superstars. The American Economic Review, 71, 5, pp. 845-858.

Schjoedt, L. (2009). Entrepreneurial Job Characteristics: An Examination of Their Effect on Entrepreneurial Satisfaction. Entrepreneurship Theory and Practice, May 2009, pp, 619-644.

Shane, S. \& Venkataraman, S. (2000). The Promise of Entrepreneurship as a Field of Research. Academy of Management Review, 25, 1, pp. 217-226.

Throsby, D. \& Hollister, V. (2003). Don't give up your day job: an economic study of professional artists in Australia. Australia Council for the Arts.

Throsby, D. (2001). Economics and Culture. Cambridge University Press, Massachusetts.

Throsby, D. (2008). Modelling the cultural industries. International Journal of Cultural Policy, 14, 3, pp.217-232.

Throsby, D. (2008). The concentric circles model of the cultural industries. Cultural Trends, 17, 3, pp. 147-164.

UNESCO. (2006). Understanding creative industries. Cultural statistics for public-policy making. France: Global Alliance for Cultural Diversity.

United Nations Conference on Trade and Development. (2004). Creative Industries and Development. Eleventh session Sao Pablo, 13-18 June 2004.

United Nations Conference on Trade and Development. (2008). Creative Economy: 2008 Report. United Nations.

Van Praag, C.M. (1999). Some classics views on Entrepreneurship. De Economist, 147, 3, pp. 311-335.

Wilson, N., \& Stokes, D. (2002). Cultural Entrepreneurs and Creating Exchange. Journal of Research in Marketing \& Entrepreneurship, 4, 2, pp. 37-52.

Recibido: 3 de agosto de 2011 Aceptado: 4 de junio de 2012 